University of Nebraska - Lincoln DigitalCommons@University of Nebraska - Lincoln

2019 Workshop: Interviewers and Their Effects

from a Total Survey Error Perspective

Sociology, Department of

$1-26-2019$

\title{
Behavior Change Techniques for Reducing Interviewer Contributions to Total Survey Error
}

Brad Edwards

Westat, bradedwards@westat.com

Follow this and additional works at: http://digitalcommons.unl.edu/sociw

Part of the Quantitative, Qualitative, Comparative, and Historical Methodologies Commons

Edwards, Brad, "Behavior Change Techniques for Reducing Interviewer Contributions to Total Survey Error" (2019). 2019 Workshop: Interviewers and Their Effects from a Total Survey Error Perspective. 1.

http://digitalcommons.unl.edu/sociw/1

This Article is brought to you for free and open access by the Sociology, Department of at DigitalCommons@University of Nebraska - Lincoln. It has been accepted for inclusion in 2019 Workshop: Interviewers and Their Effects from a Total Survey Error Perspective by an authorized administrator of DigitalCommons@University of Nebraska - Lincoln. 


\section{Behavior Change Techniques for Reducing I nterviewer Contributions to Total Survey Error}

Brad Edwards, Hanyu Sun, and Ryan Hubbard

Presented at the Symposium on Interviewer Effects and Total Survey Error, Lincoln, Nebraska February 26, 2019 


\title{
Key Words
}

\section{Talk Outline}

\author{
Background \\ Data and Methods \\ Rapid Feedback from CARI \\ Rapid Feedback of Alerts from Data \\ Impact on Key Survey Estimates \\ Conclusion
}




\section{Background}

> Most interviewer training delivered before data collection, BUT

- Most adults learn better on-the-job, just-in-time, by doing, with peers

$>$ Field interviewers work remotely in face-to-face survey operations, so very hard to see what they are doing, BUT

- Tools available now to bring field operations under much greater control

- General field interview quality can be improved with rapid feedback (verbal and written combined) of results from behavior coding of CARI recordings 


\section{Goals of This Research}

> Replicate research findings on impact of rapid feedback from CARI behavior coding on general interview quality

> Determine whether rapid feedback from CARI can impact specific interview items that are instrumental in development of key survey statistics

> Determine whether rapid feedback from automated analysis of survey data can impact data quality

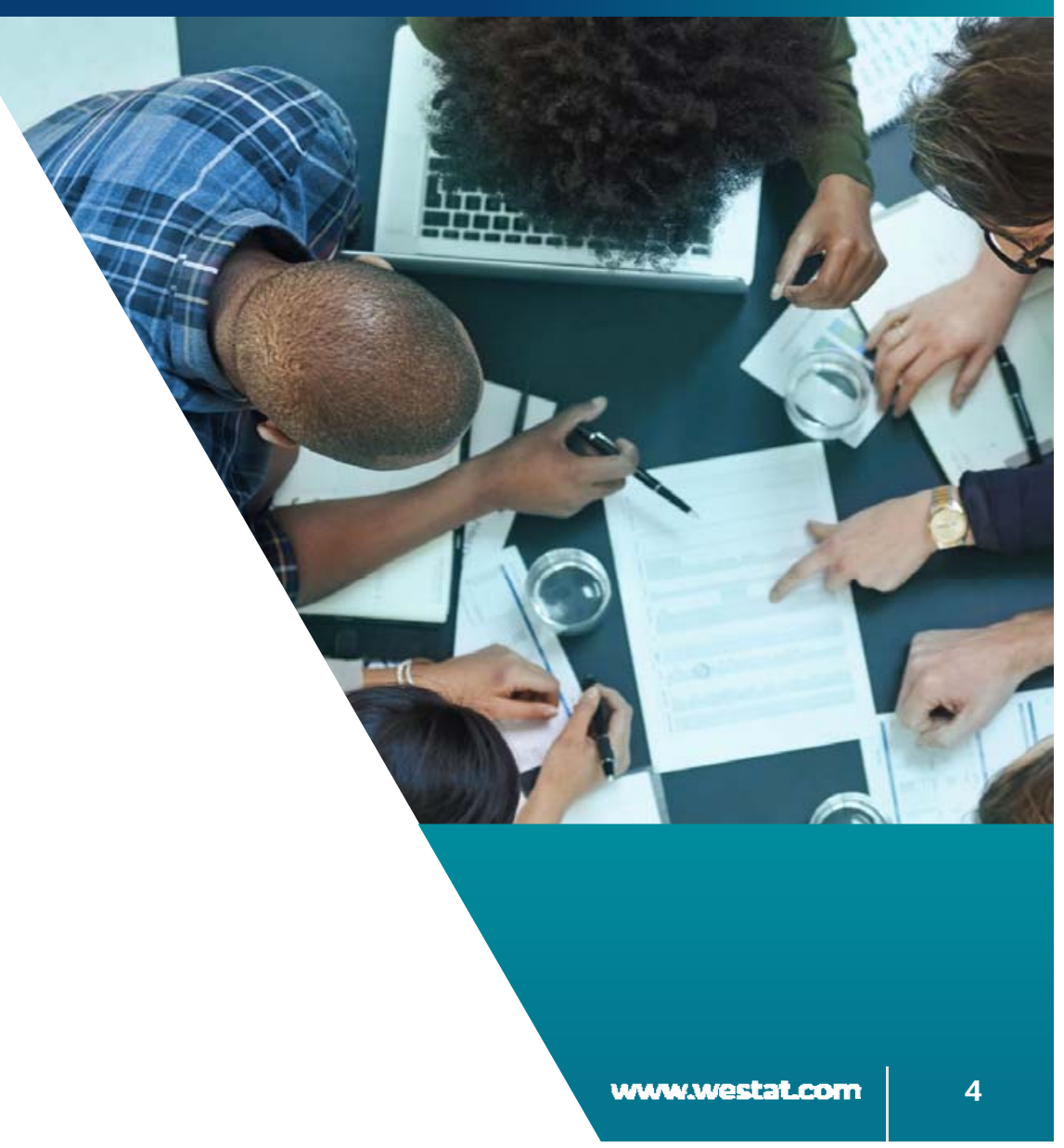




\section{Medical Expenditure Panel Survey (MEPS): Calendar Series}

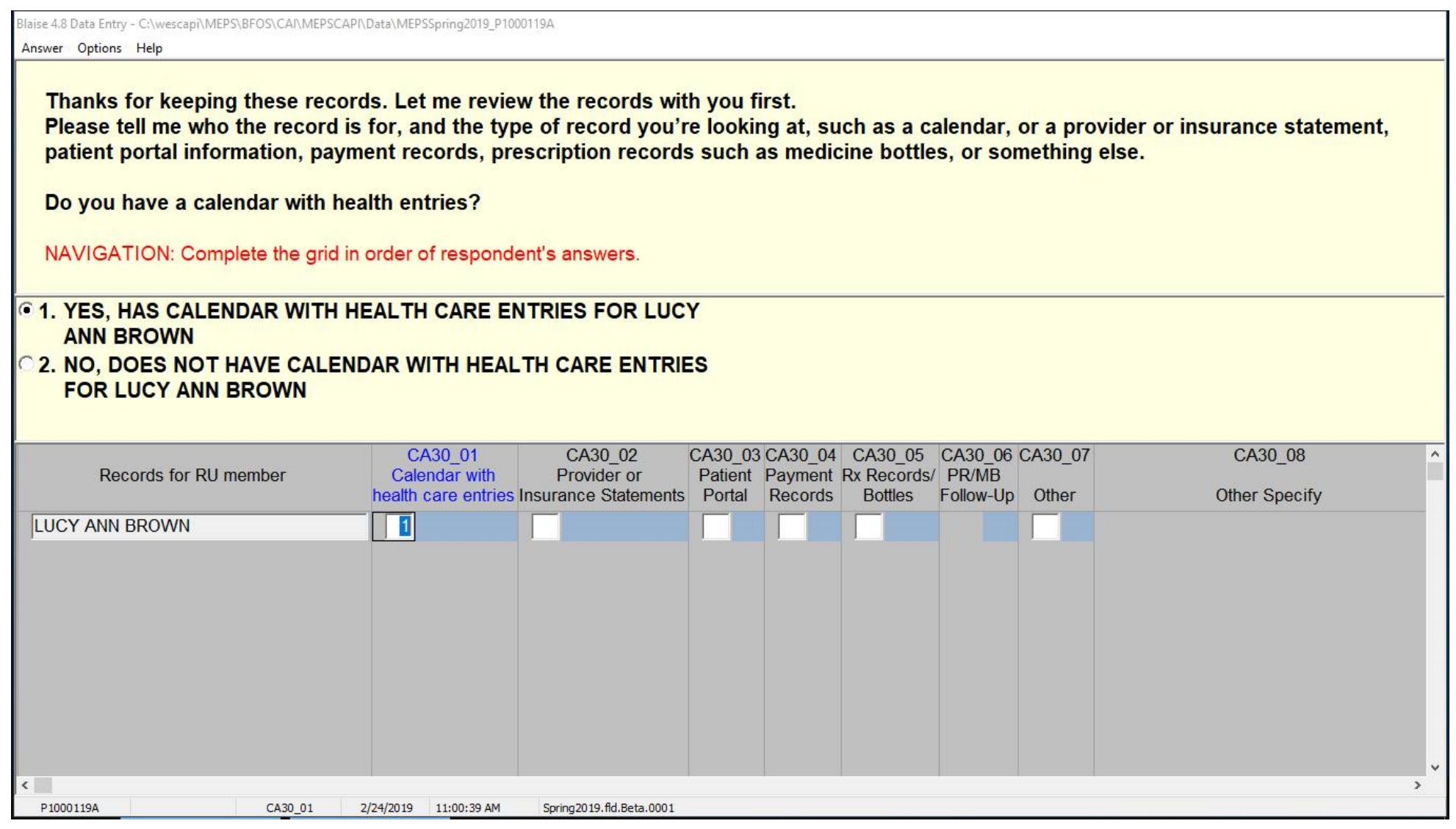




\section{MEPS: Provider Probes Series}

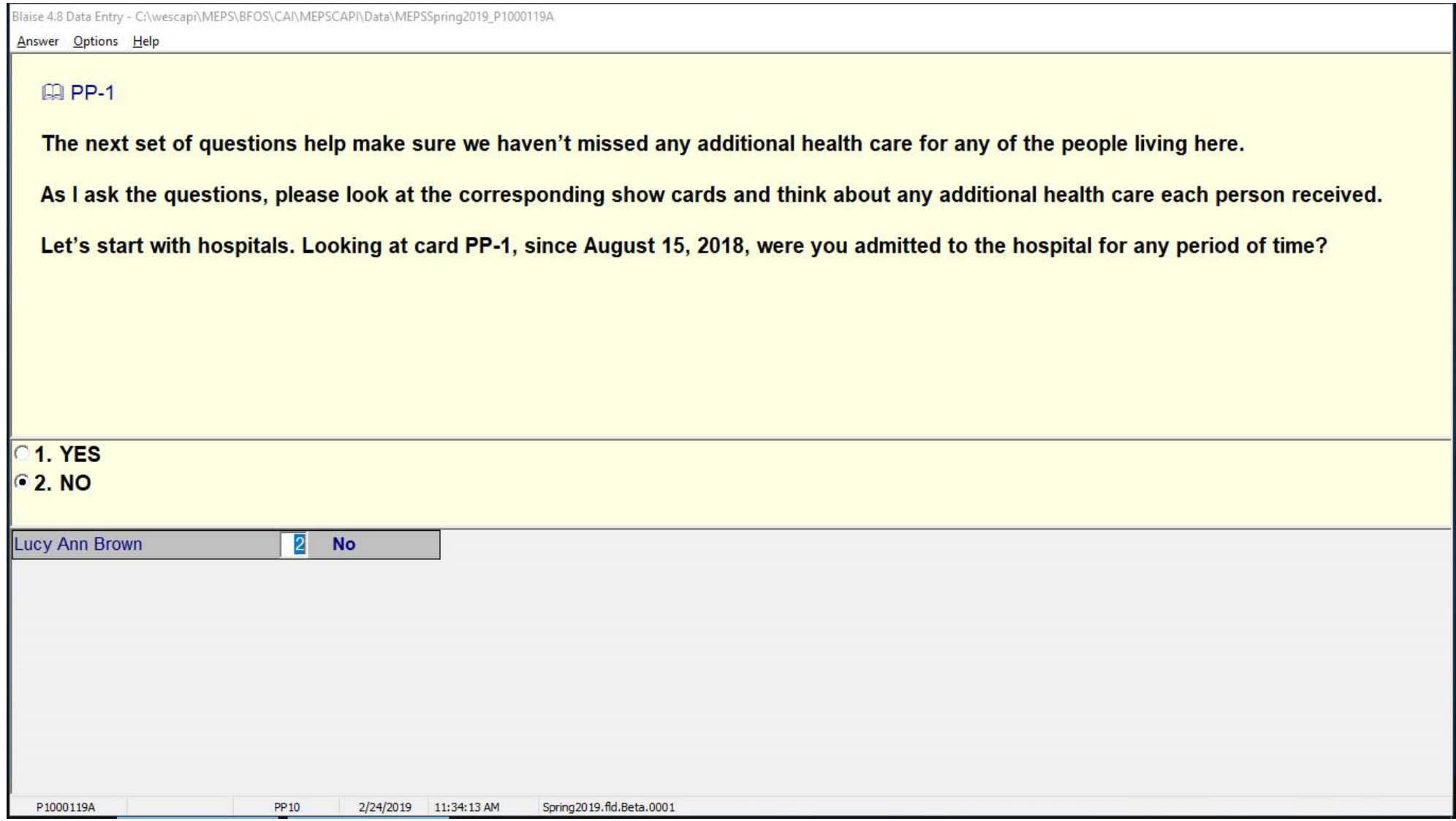




\section{CARI code Rapid Feedback Process}

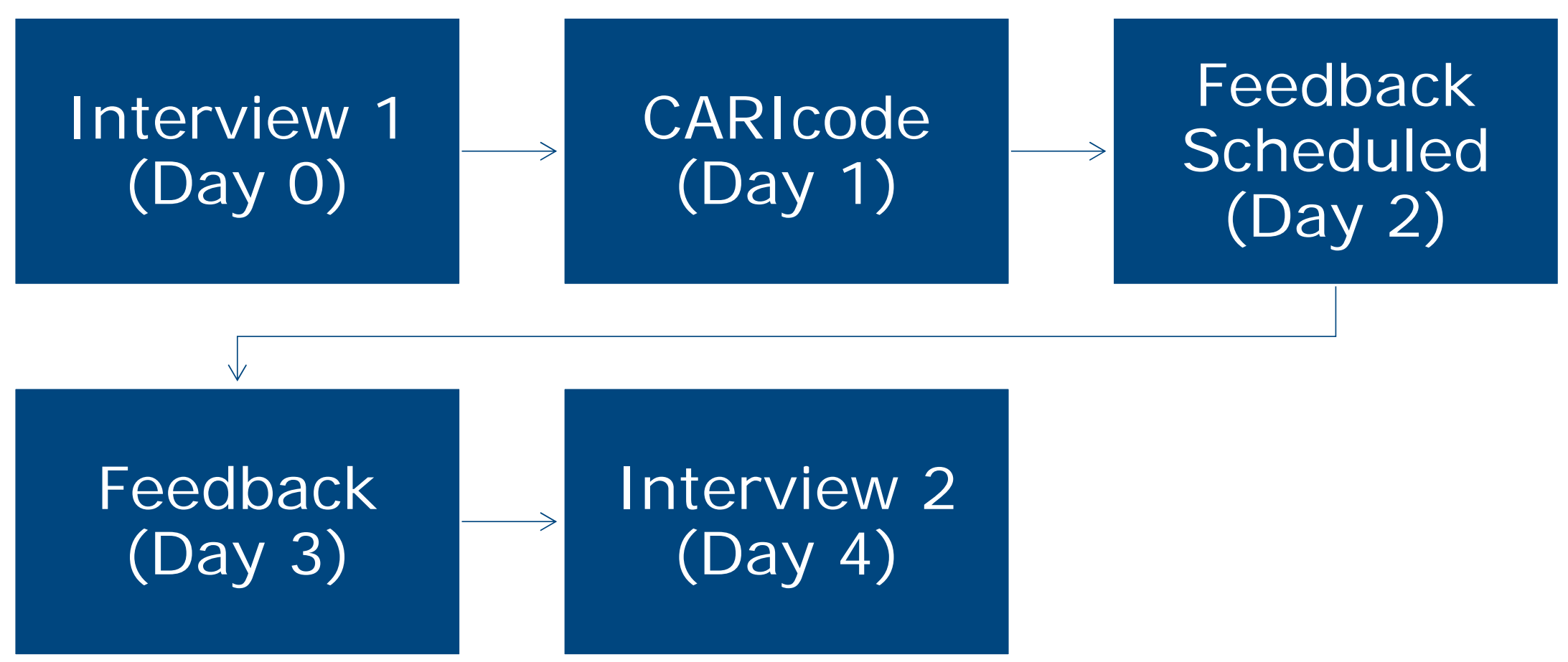




\section{Both Question Series, CARI code Results}

\begin{tabular}{|r|r|r|}
\hline $\begin{array}{r}\text { I nterviewer } \\
\text { Behavior }\end{array}$ & Before Feedback & After Feedback \\
\hline $\begin{array}{r}\text { Followed Protocol } \\
\text { Exactly }\end{array}$ & $33.4 \%$ & $43.4 \%$ \\
$\begin{array}{r}\text { (Verbatim for PP, } \\
\text { Respondent's Order } \\
\text { for CA) }\end{array}$ & $56.8 \%$ & $52.9 \%$ \\
\hline $\begin{array}{r}\text { Maintained Meaning } \\
\text { but Did Not Follow } \\
\text { Protocol Exactly }\end{array}$ & $9.8 \%$ & $3.7 \%$ \\
\hline $\begin{array}{r}\text { Meaning Not } \\
\text { Maintained }\end{array}$ & 3072 & 2187 \\
\hline $\mathrm{N}$
\end{tabular}




\section{Clarification in Feedback Session}

Verbatim vs. Meaning Not Maintained:Feedback by Interviewer Clarification

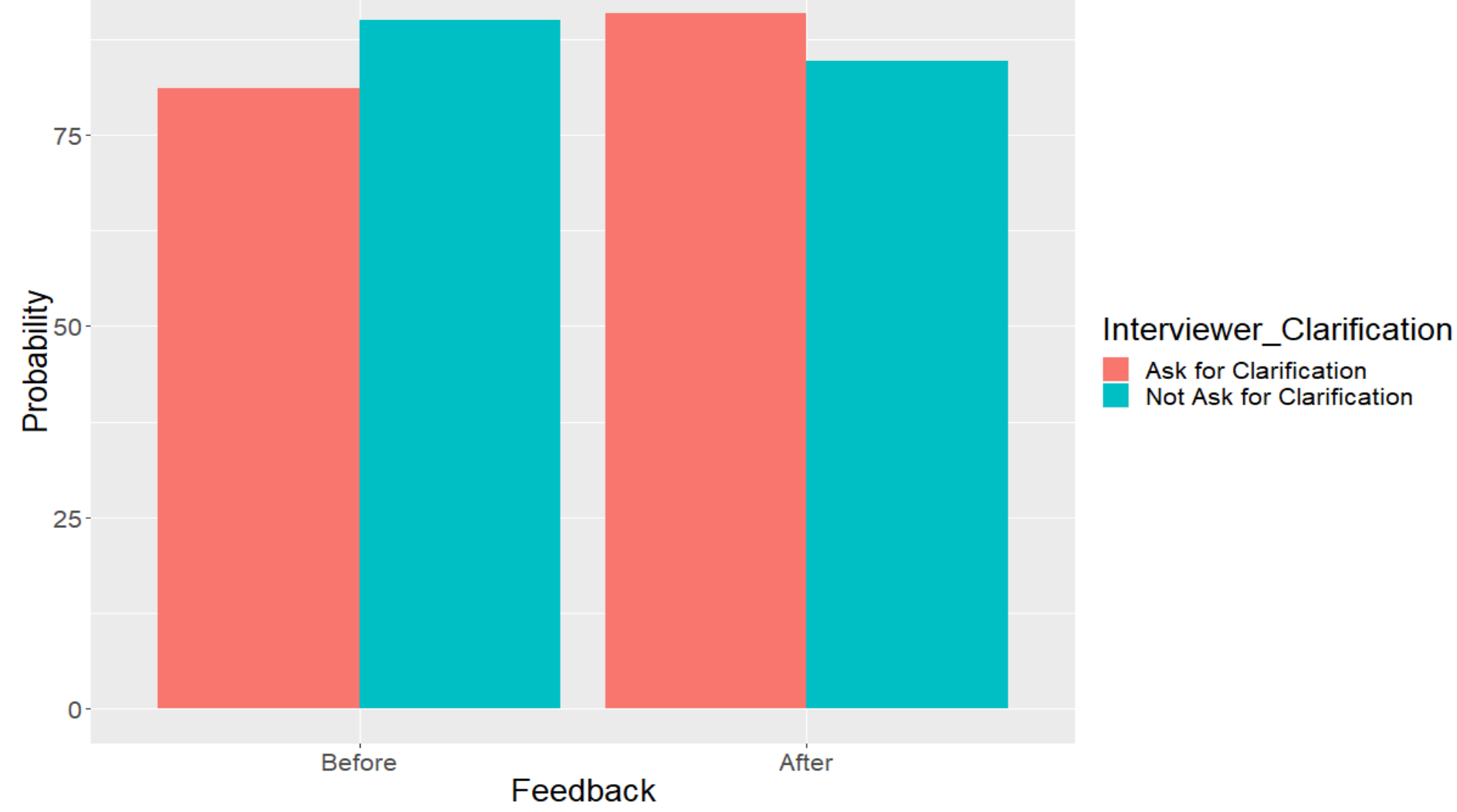




\section{Clarification Effect during Feedback Driven by Provider Probes}
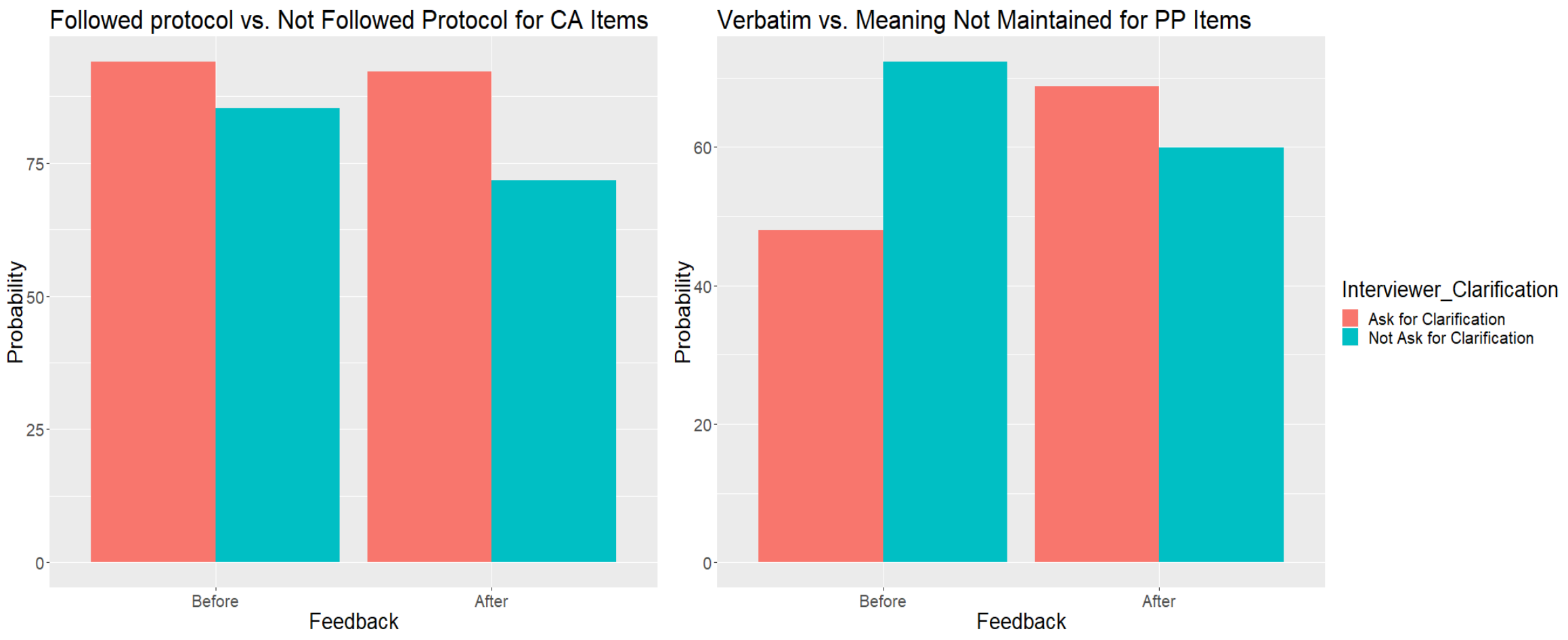


\section{Rapid Feedback: Discussion}

$>$ Interviewer experience did not explain different effects of asking clarification

$>$ CA series' flexible grid requires "offthe-grid" interviewer navigation

- perhaps even after getting clarification, some interviewers just don't get it

$>$ Nature of question content differs between 2 series

- maybe some interviewers don't believe CA makes a difference, even after getting clarification

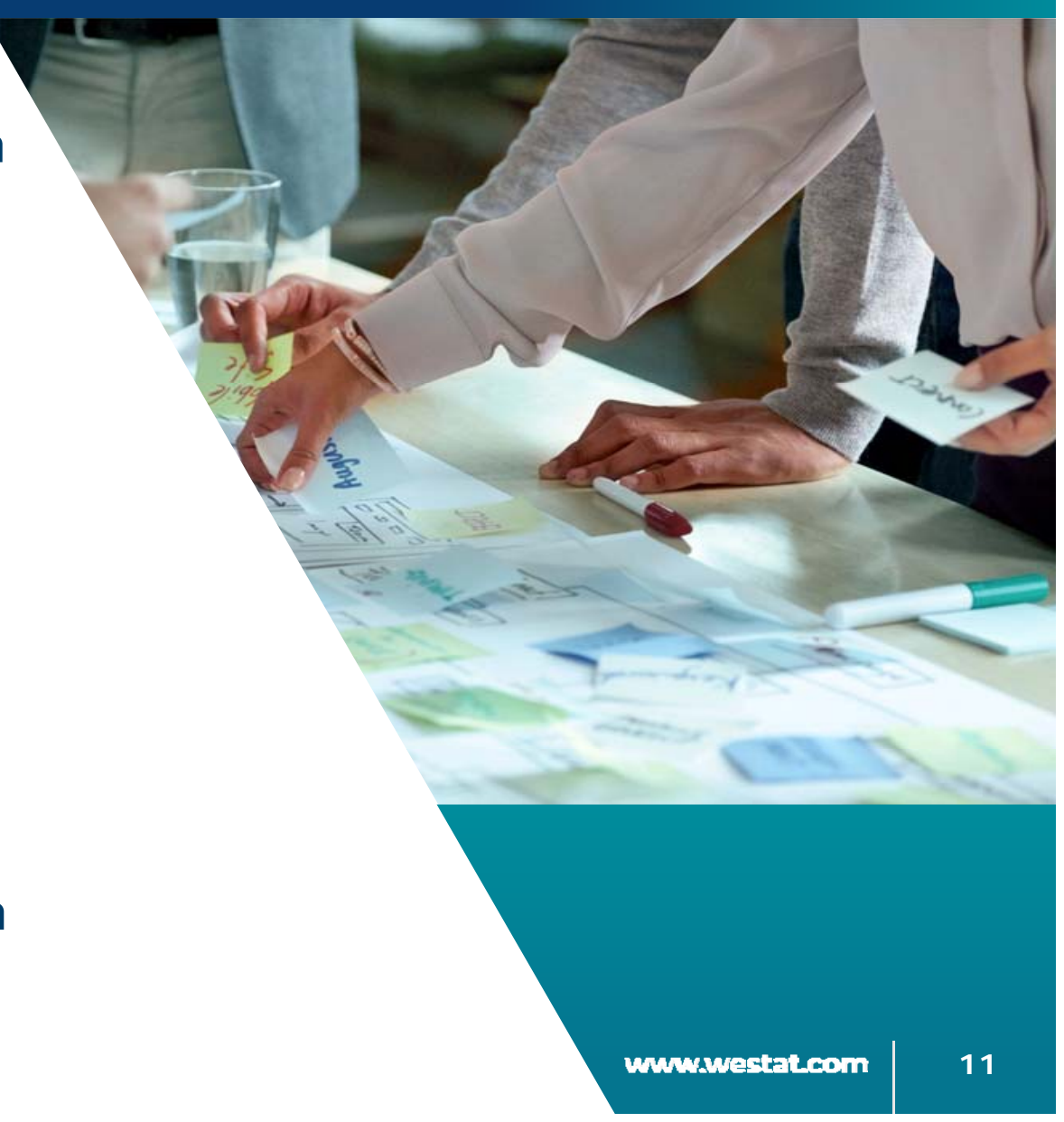




\section{Quality Alerts from Data}

$>$ Implemented through field supervisor dashboard

> Data transmitted overnight from interviewers in the field automatically checked for specific anomalies that needed immediate attention

> Anomalies popped up on supervisor dashboard the next morning

> Supervisors reviewed anomalies with interviewers and documented status in the alert section of the dashboard 


\section{Data Quality Alert Distribution}

Alert Type

Record usage (general) All medical events

Record usage Prescribed Medicines (65+)

Zero night hospital stays

(admission and discharge on same day)

Respondent under 18

Total

Westat
Count Percent $1968 \quad 84.4$

$243 \quad 10.4$

117

5.0

4

2332

100.0 


\section{Caseload-adjusted Alert Counts Over Field Period}

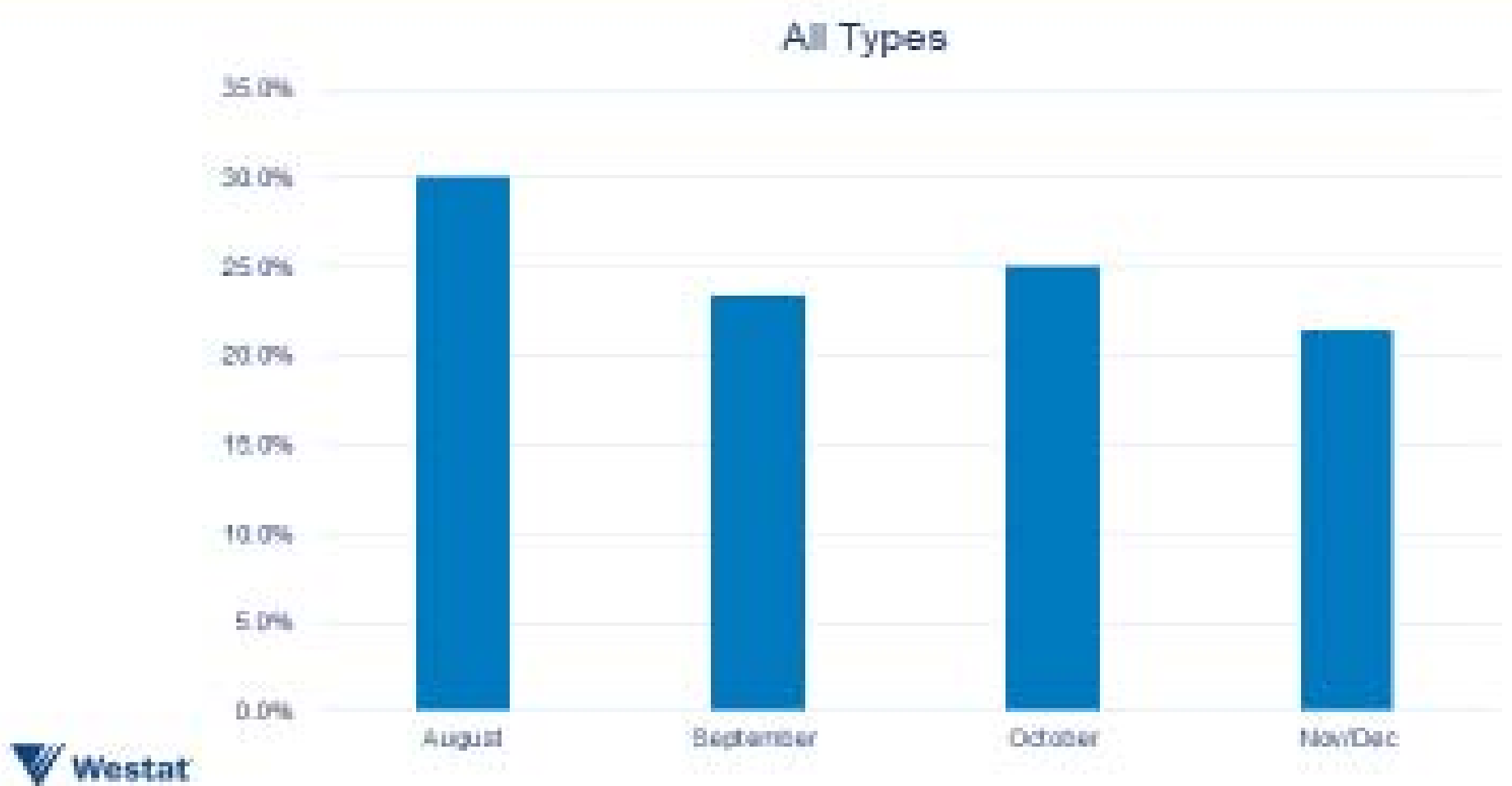




\section{Alert Occurrence Among Interviewers}

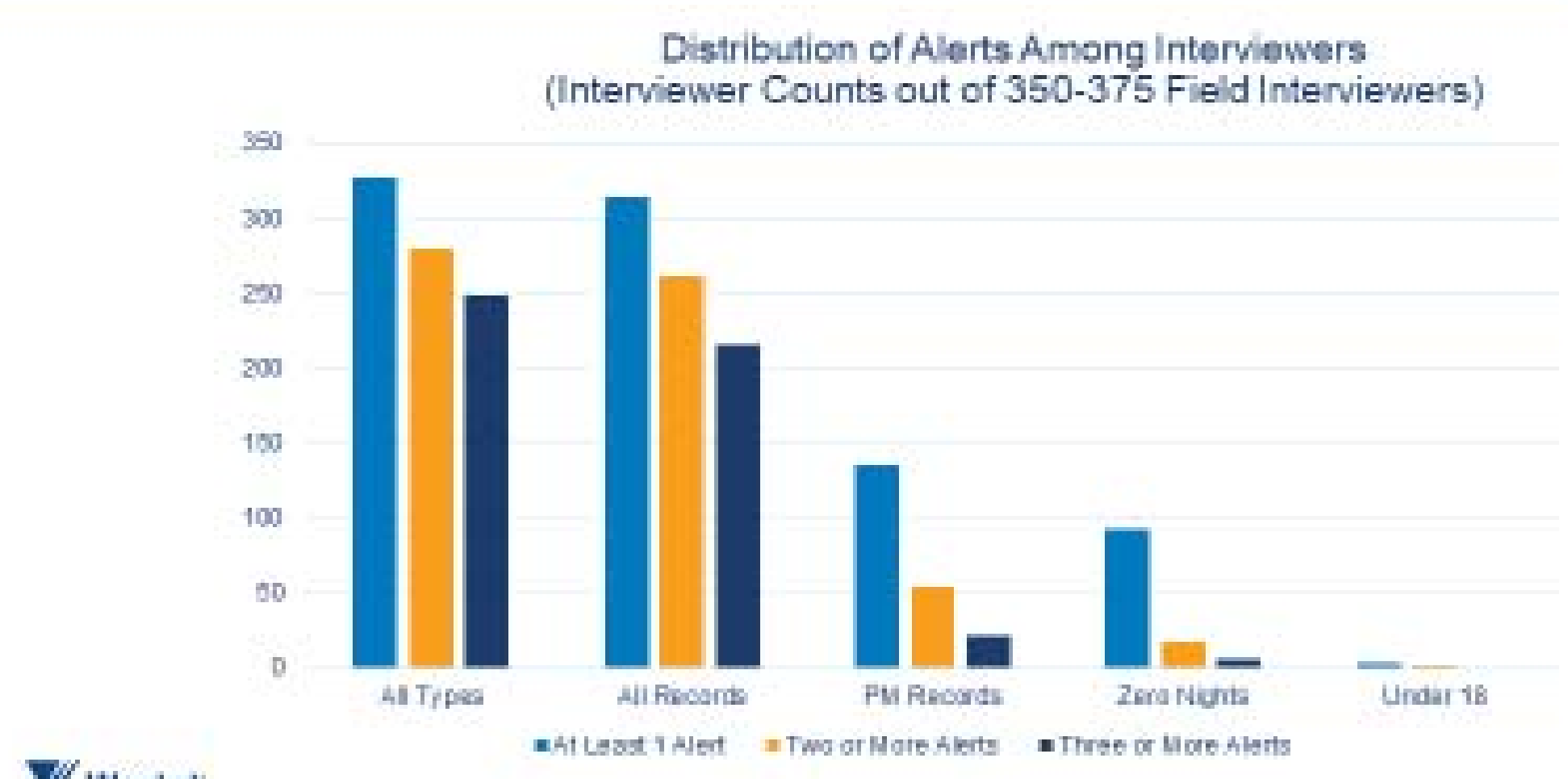




\section{Key MEPS Statistics: Rx for Older People, 2016}

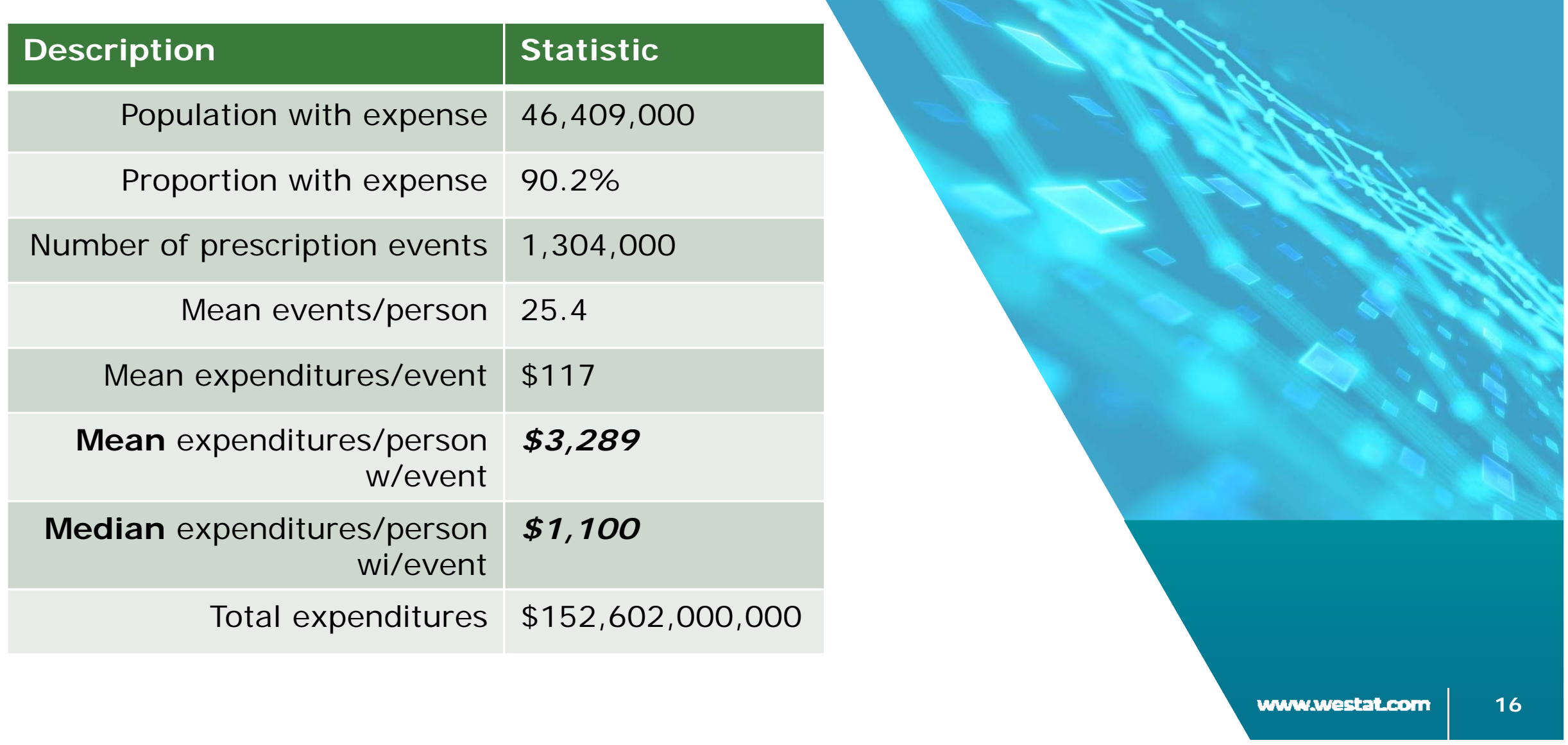




\section{Conclusion}

> Rapid feedback on techniques for asking specific questions related to key survey statistics can improve interviewer performance

> Rapid feedback on raw data collected in the interview can improve interviewer performance

$>$ Rapid feedback can be an effective form of interviewer training

$>$ Improved interviewer performance $=$ improved respondent performance

> Rapid feedback can improve the quality of key survey statistics 
Thank You

\section{Brad Edwards}

bradedwards@westat.com 